\title{
Rapid identification, capsular typing and molecular characterization of Streptococcus pneumoniae by using whole genome nanopore sequencing
}

S. Garcia-Garcia ${ }^{1 *}$, A. Perez-Arguello ${ }^{1}$, D. Henares ${ }^{1,2}$, N. Timoneda $^{1,2}$ and C. Muñoz-Almagro ${ }^{1,2,3}$

\begin{abstract}
Background: Whole genome sequencing has emerged as a useful tool for identification and molecular characterization of pathogens. MinION (Oxford Nanopore) is a real-time third generation sequencer whose portability, affordability and speed in data production make of it an attractive device for whole genome sequencing. The objective of this study is to evaluate MinION sequencer for pathogen identification and molecular characterization of Streptococcus pneumoniae isolated at a children's Hospital. Whole genome sequencing of 32 Streptococcus pneumoniae invasive isolates, previously characterized by standard methods (Quellung reaction, Multiplex PCR and Sanger-MLST), were performed. DNA was extracted using ZymoBIOMICS DNA Microprep kit. Quantification and purity of DNA was assessed by Qubit and Nanodrop, respectively. Library preparation was performed using the Rapid Barcoding Kit. Realtime workflow EPI2ME platform "What's it in my pot" was used for species identification. Fast5 sequences were converted into FASTQ by Albacore software. Reads were assembled using CANU software. PathogenWatch, genomic epidemiology and pubmlst online tools were used for capsular typing and/or whole genome-MLST profile.

Results: Rapid identification of Streptococcus pneumoniae was achieved by "What's in my pot". Capsular typing was correctly assigned with PathogenWatch in all 32 isolates at serogroup level and 24 at serotype level. Whole genomeMLST results obtained by genomic epidemiology and pubmlst were consistent with double locus variant clonal complex obtained by Sanger-MLST in 31 isolates.

Conclusion: MinION sequencer provides a rapid, cost-effective and promising pathway for performing WGS by a pocked-sized device for epidemiological purposes but improving its sequencing accuracy will make it more appealing to be used in clinical microbiology laboratories.
\end{abstract}

Keywords: Whole genome sequencing, MinION, Capsular typing, Wg-MLST, Streptococcus pneumoniae

\footnotetext{
*Correspondence: selenegarciagg@gmail.com

'Institut de Recerca Sant Joan de Deu, Hospital Sant Joan de Deu, Barcelona, Spain

Full list of author information is available at the end of the article
}

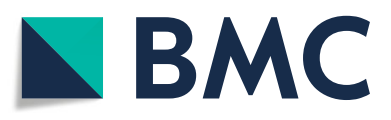

(- The Author(s). 2020 Open Access This article is licensed under a Creative Commons Attribution 4.0 International License, which permits use, sharing, adaptation, distribution and reproduction in any medium or format, as long as you give appropriate credit to the original author(s) and the source, provide a link to the Creative Commons licence, and indicate if changes were made. The images or other third party material in this article are included in the article's Creative Commons licence, unless indicated otherwise in a credit line to the material. If material is not included in the article's Creative Commons licence and your intended use is not permitted by statutory regulation or exceeds the permitted use, you will need to obtain permission directly from the copyright holder. To view a copy of this licence, visit http://creativecommons.org/licenses/by/4.0/. The Creative Commons Public Domain Dedication waiver (http://creativecommons.org/publicdomain/zero/1.0/) applies to the data made available in this article, unless otherwise stated in a credit line to the data. 


\section{Background}

Streptococcus pneumoniae is responsible for causing disease especially in children younger than 5 years and the elderly. It is associated with high morbidity and mortality worldwide [1]. Updated regional mortality estimates were published by WHO for 2008, estimating 541,000 pneumococcal deaths in children that year [2]. S.pneumoniae is a highly adaptable microorganism evolving continuously. The replacement phenomenon against vaccine strategies, referring to the expansion of non-vaccine serotypes as a result of the removal from the population of vaccine types, should be surveillance with a rapid and accurate diagnosis and molecular characterization. Traditional methods for molecular epidemiology have several limitations including a possible untimely delivery of results [3]. A conventional S.pneumoniae typing system is based on multi locus sequence typing (MLST). MLST is a procedure for characterizing isolates using the partial sequence of seven well-conserved housekeeping genes by Sanger technology. A sequence type (ST) is assigned by comparing each gene against other isolate profiles in the public MLST reference database (https://pubmlst.org/). STs are grouped into clonal complexes by their similarity to a central allelic profile [4]. Using conventional Sanger sequencing approaches, MLST turn out a laborious technique, expensive and difficult to perform at scale.

To date, at least 100 pneumococcal polysaccharide capsule types have been described [5]. The ability of pneumococci to cause disease is directly related to the polysaccharide capsule, the major virulence factor. Pneumococci can be classified into different serotypes according to their capsule type using conventional serotyping techniques. On the one hand multiplex PCR combined with both fragment analysis and automated fluorescent capillary electrophoresis. On the other hand, the gold standard Quellung reaction to complete serotyping [6].

Next generation sequencing technology provides lots of information about species, serovar, virulence, pathogenicity, antimicrobial resistance in just one sequencing run [7]. Whole genome sequencing (WGS) is evolving as a routine practice as a diagnostic. The affordable cost and the reduced turnaround time in comparison to standard methods are achieving a viable and promising technology in diagnostic. NGS is characterized by higher throughput, increased read lengths, reductions in sequencing time and low overall cost when compared to conventional DNA sequencing methods [8]. The main extra feature of third generation sequencing, which includes MinION sequencer, is the long-read length. In 2014, Oxford Nanopore Technologies launched MinION, the only pocket-sized portable real-time device for DNA and RNA sequencing. It is the smallest sequencing device available. It plugs directly into a PC or laptop via USB cable. Low hardware requirements mean a standard computer is sufficient for sequencing. Nanopore-based sequencing relies on changes in the ionic current of nucleotides as a single DNA molecule passes through artificial nanopores as a single DNA molecule [9] [10]. Nevertheless, the high error rate exhibited by MinION sequencer has limited its ability to compete with existing sequencing technologies but software developments are continuously increasing base call accuracy [11].

The work described in this study is meant to provide information on the state of MinION sequencer for assessing its capabilities for pathogen identification and molecular characterization of S.pneumoniae isolated at a children's Hospital.

\section{Results}

\section{Comparison between Nanopore technology and conventional methods}

A rapid and correct identification of all 32 S.pneumoniae invasive strains was achieved by WIMP workflow in only $5 \mathrm{~min}$, meanwhile at least two days are required by traditional bacterial identification. WGS took around $3 \mathrm{~h}$. Table 1 includes the length of time taken for each step comparing Nanopore technology and conventional microbiology approach.

Regarding prices of both workflows, the cost per sample characterized by MinION is about $65.11 €$, meanwhile by Sanger is roughly $53.40 €$.

As depicted in Table 2, when comparing MLST derived from Sanger or Nanopore data, a concordance of $16 \%$ was achieved in those strains that shared seven out of seven genes (ST). The concordance at SLV and DLV level was 56 and $25 \%$, respectively. A match of four out of seven genes (QLV) was solely obtained in one strain (3\%). Wg-MLST results obtained by genomic epidemiology and pubmlst online tools were consistent with at least DLV definition obtained by Sanger-MLST in 31/32 S.pneumoniae invasive strains. Capsular typing was correctly assigned with PathogenWatch in all 32 isolates at serogroup level and 24 at serotype level. Limitations in the resolution of genotypic inference of serotype are depicted in Table 3.

Conventional methodology refers to multiplex PCR combined with fragment analysis and automated fluorescent capillary electrophoresis and Quellung reaction, as explained previously in the methods.

\section{Discussion}

In this study we have carried out an evaluation panel of 32 isolates including pathogen identification and pneumococcal clonal characterization. The whole-genome sequencing turnaround time, from DNA extraction and library preparation to sequencing and data analysis, is roughly 2 days. Nevertheless, it comprehensively identified pathogens at species level in just $5 \mathrm{~min}$. Although there is an increasing trend of microbiologist using MALDI-TOF for bacterial identification in clinical settings, acquiring it requires a 
Table 1 MinlON and Sanger workflow times comparison

\begin{tabular}{|c|c|c|}
\hline Steps & MinION workflow & Sanger workflow \\
\hline Sample preparation & \multicolumn{2}{|c|}{$\begin{array}{l}\text { Strains cultured overnight } \\
\text { and harvested the following day }\end{array}$} \\
\hline DNA extraction & $2 \mathrm{~h}$ & $40 \mathrm{~min}$ \\
\hline Preparation for sequencing & $1 \mathrm{~h}$ & $8 \mathrm{~h} 15 \mathrm{~min}$ \\
\hline Sequencing & 5 min-3 $h^{*}$ & $6 \mathrm{~h}$ \\
\hline Data analysis & $1 \mathrm{~h}$ & $2 \mathrm{~h}$ \\
\hline Total & $4 \mathrm{~h} 5 \mathrm{~min}-7 \mathrm{~h}$ & 16 h45min \\
\hline
\end{tabular}

Clarifications for Sanger workflow: a) "Preparation for sequencing" step includes four different steps which make it more laborious [amplification (3h30min), 1st purification ( $1 \mathrm{~h} 15 \mathrm{~min})$, sequence reaction ( $3 \mathrm{~h}$ ) and 2 nd purification $(30 \mathrm{~min})]$; b) "Sequencing" step only includes the seven housekeeping genes: aroE, gdh, gki, recP, spi, xpt and ddl

"Five minutes are enough for identification at species level and at least $3 \mathrm{~h}$ are required to obtain whole genome data. However, we always initiated a standard $18 \mathrm{~h}$ sequencing procedure

Abbreviations: NA not available, WGS whole-genome sequencing

high economic investment not affordable for everyone. It is a good and reliable choice but it is not still available in too many laboratories. The sequencing approach using MinION, whereby results are generated and analysed in real time, has the potential to be much faster than Sanger technology. NGS technologies are increasingly being integrated into patient care and clinical management.

Until now Sanger sequencing has been the gold standard only for genotyping not serotyping in clinical laboratories serving as the conventional method whereby NGS data should be compared and validated [12]. WGS is a revolutionary technology that is increasingly replacing traditional clinical diagnostics. It has emerged as a useful tool for identification and molecular characterization of pathogens. However, based on the low concordance in MLST between Sanger and Nanopore data, MinION is not accurate enough to be completely replaced by conventional methods for molecular epidemiology in surveillance programs. Nevertheless, since WGS is becoming increasingly ubiquitous, conventional MLST by Sanger sequencing concept has been extended to include, apart from 7 housekeeping genes, many hundreds or even thousands of loci coined wgMLST [13].

Table 2 S. pneumoniae clonal characterization by MinION

\begin{tabular}{ll}
\hline $\begin{array}{l}\text { Sequence type or clonal } \\
\text { complex designation }\end{array}$ & $\begin{array}{l}\text { \% of } \text { S. pneumoniae strains } \\
\text { corresponding to each designation }\end{array}$ \\
\hline ST & $16 \%(5 / 32)$ \\
SLV & $56 \%(18 / 32)$ \\
DLV & $25 \%(8 / 32)$ \\
QLV & $3 \%(1 / 32)$ \\
\hline
\end{tabular}

To obtain the \% of S. pneumoniae strains corresponding to each designation, MinlON results were compared to those obtained by Sanger sequencing. A total of 32 invasive pneumococcal strains were analyzed. Wg-MLST results were achieved in $3 \mathrm{~h}$

Abbreviations: ST Sequence Type, SLV Single Locus Variantm, DLV, Double Locus Variant, QLV Quadruple Locus Variant
Table 3 Serotype comparison between conventional methodology and PathogenWatch online tool

\begin{tabular}{ll}
\hline $\begin{array}{l}\text { Serotype by conventional } \\
\text { methodology }\end{array}$ & $\begin{array}{l}\text { Serotype by MinION } \\
\text { (PathogenWatch) }\end{array}$ \\
\hline $6 \mathrm{C}$ & $6 \mathrm{D}$ \\
$9 \mathrm{~V}$ & $9 \mathrm{~A}$ \\
$9 \mathrm{~V}$ & $9 \mathrm{~A}$ \\
$9 \mathrm{~V}$ & $9 \mathrm{~A}$ \\
$18 \mathrm{C}$ & $18 \mathrm{~B}$ \\
$24 \mathrm{~F}$ & Serogroup 24 \\
$24 \mathrm{~F}$ & Serogroup 24 \\
$24 \mathrm{~F}$ & Serogroup 24 \\
\hline
\end{tabular}

In addition, its capability to produce longer reads and its low price compared to other sequencers make of it an attractive device for WGS. The major advantages are the price, the speed in data production, which would make it appropriate in a hospital for rapid diagnosis, and the ability to sequence a single isolate rather than wait to fill a lane of an Illumina sequencer. Regarding the price, as mentioned elsewhere, due to a single flow cell can be used until twice, the cost per 2 runs (12 samples per run; 24 samples) is around 1562.66€; 65.11€ per sample. Alike, the cost per sample characterized by Sanger workflow is roughly $53.40 €$. However, several considerations have to be taken into account, namely MinION provides information about the entire pathogen's genome, meanwhile Sanger characterization is only focus on seven housekeeping genes. Moreover, the time invested in preparation for sequencing is vastly higher in Sanger workflow than that of MinION. The same thing happens with the sequencing time, which is higher 2fold with Sanger. For these reasons, the total invested time for Sanger workflow is increased 4-fold. Nevertheless, as a limitation of the study, three serogroup 24 were not correctly matched between conventional and MinION methodologies. This event could be explained due to the genome-based method can not subtype serogroup 24. This is not a discrepancy but a limitation in the genome-based serotyping method [14].

Although the approach has great potential, challenges remain. Firstly, the main limitation of MinION sequencing is its lower read accuracy when compared to short-read technologies. While Illumina's quality PHRED score is about 30, MinION moves around 10 (Supplementary table) [8]. Because of that, MinION still has not moved into routine clinical practice. Secondly, strains were collected from our laboratory biobank where they are stored once identified and characterized. Thus, isolates did not proceed directly from clinical samples. Its issue is referring to low-input procedures that notably reduce the DNA requirement for nanopore sequencing. Thirdly, we tested up to 24 strains per flow cell. It might induce to cross 
contamination between two consecutive runs. However, the price per sequenced strain undoubtedly decreases as a same flow cell is used twice. Finally, with the automation of bioinformatic pipelines, this method could become very attractive for monitoring invasive S.pneumoniae strains. The great amount of information generated with WGS can be easily valued with, for example, the analysis of molecular evolution of the isolates, the identification of putative vaccine targets in addition to the detection of antibiotic resistance and virulence genes [15].

\section{Conclusion}

In conclusion, MinION sequencer provides a rapid, costeffective and promising pathway for performing WGS by a pocked-sized device for epidemiological purposes but improving its sequencing accuracy will make it more appealing to be used in clinical microbiology laboratories.

\section{Methods}

\section{Study setting and design}

The study was conducted at University Hospital Sant Joan de Deu Barcelona (Spain) during 2018. The setting study is a 318-bedsize reference university children's hospital that attends a paediatric reference population of approximately 300,000 subjects. In 2009, the Molecular Microbiology Laboratory was designated by the government of Catalonia (Spain) as the Catalan support laboratory for molecular surveillance of invasive pneumococcal disease (IPD). Catalan Hospitals are invited, not forced, to send invasive pneumococcal strains. For the present study, neither ethics approval nor informed consent to participate were requested as it is a proof of concept study in which samples were duly anonymized.

\section{Microbiological methods}

An evaluation panel including a total of 32 invasive pneumococcal isolates recovered from sterile fluid samples (blood, cerebrospinal fluid and pleural fluid) was performed. Samples were obtained from pure cultures around $10^{8} \mathrm{UCF} / \mathrm{ml}$ corresponding to $0.5 \mathrm{McF}$ arland in order to obtain $400 \mathrm{ng}$, the required input DNA for sequencing. All 32 strains were stored, in $1.5 \mathrm{~mL}$ microcentrifuge tubes with screw cap, with skimmed milk as a growth media preserved and conserved in the biobank facilities of the University Hospital Sant Joan de Deu. These strains had previously been identified and characterized by the Molecular Microbiology Laboratory with conventional methods (Quellung reaction, Multiplex PCR and SangerMLST) before being analysed by MinION.

\section{Conventional methods}

\section{Microbiological identification}

All pneumococcal invasive isolates were cultured on blood agar plates (Columbia agar supplemented with 5\% sheep blood; bioMérieux) and were identified by standard microbiological methods including optochin sensitivity test and an antigenic test.

\section{Serotype analysis}

Identification of capsular pneumococcal serotypes was performed using multiplex PCR combined with fragment analysis and automated fluorescent capillary electrophoresis. This technique has been used since 2010 and allows the detection of 40 serotypes/serogroups: $(1,2,3,4,5$, 6A/6B, 6C, 7F/7A, 7C/(7B/40), 8, 9 V/9A, $9 \mathrm{~N} / 9 \mathrm{~L}, 10 \mathrm{~A}$, $10 \mathrm{~F} /(10 \mathrm{C} / 33 \mathrm{C}), 11 \mathrm{~A} / 11 \mathrm{D} / 11 \mathrm{~F}, 12 \mathrm{~F} /(12 \mathrm{~A} / 44 / 46), 13,14$, $15 \mathrm{~A} / 15 \mathrm{~F}, 15 \mathrm{~B} / 15 \mathrm{C}, 16 \mathrm{~F}, 17 \mathrm{~F}, 18 /(18 \mathrm{~A} / 18 \mathrm{~B} / 18 \mathrm{C} / 18 \mathrm{~F})$, 19A, 19F, 20, 21, 22F/22A, 23A, 23B, 23F, 24/(24A/24B/ $24 \mathrm{~F}), 31,33 \mathrm{~F} /(33 \mathrm{~A} / 37), 34,35 \mathrm{~A} /(35 \mathrm{C} / 42), 35 \mathrm{~B}, 35 \mathrm{~F} / 47 \mathrm{~F}$, $38 / 25 \mathrm{~F}$ and 39) [16]. All strains were also sent to the $\mathrm{Na}-$ tional Pneumococcus Reference Center of Majadahonda, Madrid, Spain, to complete serotyping by Quellung reaction and determine antimicrobial susceptibility study.

\section{Clonal analysis}

ST analysis was performed with MLST. MLST involves PCR amplification of seven housekeeping genes followed by Sanger DNA sequencing [17] [6]. Allele assignation and ST were carried out using the software at the pneumococcal web page http://pubmlst.org/spneumoniae/. STs are grouped into clonal complexes (CCs) by their similarity to a central allelic profile [18]. STs that shared 6 out of 7 allelic variants (SLV, single locus variants) and 5 out of 7 allelic variants (DLV, double locus variants) were considered a CC.

\section{MinION pipeline}

Sample preparation

Strains were collected from our laboratory biobank. They were cultured overnight and harvested the following day.

\section{DNA extraction}

DNA was extracted according to manufacturer instructions of ZymoBIOMICS DNA Microprep kit (ZYMO RESEARCH) with minor changes according to the instructions of the manufacturer. Extraction procedure required about $2 \mathrm{~h}$ ' time per 12 samples. This is a columnbased system for total nucleic acid extraction. Quantification of double strand DNA (dsDNA) through a fluorimetric assay was performed using Qubit (Life Technologies, Carlsbad, California, US) and the High Sensitivity (HS) dsDNA Assay Kit under manufacturer's conditions. Purity was assessed through NanoDrop ${ }^{\mathrm{Tm}}$ Spectrophotometer (Thermo Fisher Scientific, Waltham Massachusetts, US).

\section{Library preparation and sequencing}

Library preparation was performed using the Rapid Barcoding Kit (SQK-RBK004) provided by Oxford Nanopore 
Technologies with an R9 flow cell (FLO-MIN106). The procedure took approximately $1 \mathrm{~h}$ per 12 samples. MinION libraries were created with $400 \mathrm{ng}$ of total genomic DNA for each sample with an adjusted volume of $7.5 \mu \mathrm{L}$ Nuclease-free water. A clean-up step using Agencourt AMPure XP beads (Beckman coulter, Munich, Germany) at $1 \mathrm{X}$ concentration was used to discard short fragments as recommended by the manufacturer. DNA quantity was assessed using Qubit fluorimeter. Promega calculator (https://www.promega.es/resources/tools/biomath/) was used to convert $\mu \mathrm{g}$ dsDNA into pmol. This parameter must fall between $0.2-0.25 \mathrm{pmol}$, as specified in the protocol kit. This amount refers to the prepared library that must be loaded into the MinION flow cell. Once the library has been loaded, we initiated a standard $18 \mathrm{~h}$ sequencing procedure using the MinKNOW software, and by using the live basecalling feature.

\section{Data analysis}

During sequencing or once MinION finishes running, the data set was analysed by the real-time workflow provided by Nanopore EPI2ME platform "What's in my pot?" (WIMP), which assigns taxonomy by comparing read sequences against a database. After sequencing, fast 5 sequences were converted into FASTQ by using Albacore software. Reads were assembled using CANU for clonal analysis [19]. Once assembled, allelic variants were obtained through www.genomicepidemiology.org website. Finally, ST analysis was performed using the software at the pneumococcal web page http://pubmlst. org/spneumoniae/.

For MLST assignment from whole genome data (wgMLST), contigs generated by CANU were uploaded on PathogenWatch online tool (https://pathogen.watch/ ), a global platform for genomic surveillance. Conventional MLST by Sanger sequencing, which is basically only focus on 7 housekeeping genes, can be extended to wgMLST by which many more loci are considered.

\section{Raw data}

No administrative permissions were required to access the raw data as no human DNA was analyzed. Only bacterial DNA from S.pneumoniae strains was analyzed.

\section{Supplementary Information}

The online version contains supplementary material available at https://doi. org/10.1186/s12866-020-02032-x.

Additional file 1 Supplementary Table. Statistics description of raw

read sequences obtained by MinlON.

\section{Abbreviations}

S.pneumoniae: Streptococcus pneumoniae; MLST: Multi Locus Sequence Typing; ST: Sequence Type; WGS: Whole Genome Sequencing; IPD: Invasive Pneumococcal Disease; MIC: Minimum Inhibitory Concentrations;
EUCAST: European Committee on Antimicrobial Susceptibility Testing; CC: Clonal Complex; SLV: Single Locus Variant; DLV: Double Locus Variant: dsDNA: Double strand DNA; HS: High Sensitivity; WIMP: What's In My Pot; QLV: Quadruple Locus Variant

\section{Acknowledgments}

The authors would acknowledge Dr. Olga Francino of Autonomous University of Barcelona.

\section{Authors' contributions}

SGG performed laboratory test and acquired test result data, analysed and interpreted data, prepared the first draft manuscript and revised and approved the final version of the manuscript; APA performed laboratory test and acquired test result data and revised and approved the final version of the manuscript, DH interpreted data and revised and approved the final version of the manuscript; NT analysed and interpreted data and revised and approved the final version of the manuscript; CMA defined the study design, supervised the study, interpreted data and revised and approved the final version of the manuscript. All authors have read and approved the manuscript.

\section{Funding}

This work was supported by the research grant PI19/00104 from Instituto de Salud Carlos III, co-financed by the European Regional Development Fund (ERDF). The funding body did not have any role in the design of the study, in the collection, analysis, and interpretation of data, or in writing the manuscript.

\section{Availability of data and materials}

Raw metagenomic reads were submitted to NCBI Sequence Read Archive (SRA), under the bioproject number PRJNA643905.

Ethics approval and consent to participate

Not applicable.

\section{Consent for publication}

Not applicable.

\section{Competing interests}

CMA reports a research grant of Pfizer laboratories to our institution outside the submitted work.

\section{Author details}

${ }^{1}$ Institut de Recerca Sant Joan de Deu, Hospital Sant Joan de Deu, Barcelona, Spain. ${ }^{2}$ CIBER de Epidemiologia y Salud Publica (CIBERESP), Instituto de Salud Carlos III, Madrid, Spain. ${ }^{3}$ Departament de Medicina, Universitat Internacional de Catalunya, Barcelona, Spain.

Received: 13 April 2020 Accepted: 3 November 2020

Published online: 13 November 2020

\section{References}

1. World Health Organization (WHO). Weekly epidemiological record. WHO. 2007. Available from: http://www.who.int/wer/2007/wer8212.pdf?ua=1.

2. Wahl B, O'Brien $\mathrm{KL}$, Greenbaum A, Majumder A, Liu L, Chu Y, et al. Burden of Streptococcus pneumoniae and Haemophilus influenzae type $b$ disease in children in the era of conjugate vaccines: global, regional, and national estimates for 2000-15. Lancet Glob Heal. 2018;6:e744-57.

3. Eybpoosh S, Haghdoost AA, Mostafavi E, Bahrampour A, Azadmanesh K, Zolala F. Molecular epidemiology of infectious diseases. Electron Physician. 2017:9:5149-58.

4. Jolley KA, Bray JE, Maiden MCJ. Open-access bacterial population genomics: BIGSdb software, the PubMLST.org website and their applications. Wellcome Open Res 2018;3:1-20

5. Ganaie F, Saad JS, McGee L, van Tonder AJ, Bentley SD, Lo SW, et al. A new pneumococcal capsule type, 10D, is the 100th serotype and has a large cps fragment from an oral streptococcus. MBio. 2020;11:1-15.

6. Selva L, Del Amo E, Brotons P, Muñoz-Almagro C. Rapid and easy identification of capsular serotypes of Streptococcus pneumoniae by use of fragment analysis by automated fluorescence-based capillary electrophoresis. J Clin Microbiol. 2012;50:3451-7. 
7. Ibrahim GM, Morin PM. Salmonella serotyping using whole genome sequencing. Front Microbiol. 2018;9:1-8.

8. Wang $Y$, Yang $Q$, Wang $Z$. The evolution of nanopore sequencing. Front Genet. 2014;5:1-20.

9. Pareek CS, Smoczynski R, Tretyn A. Sequencing technologies and genome sequencing. J Appl Genet. 2011;52:413-35.

10. Bowden R, Davies RW, Heger A, Pagnamenta AT, de Cesare M, Oikkonen LE, et al. Sequencing of human genomes with nanopore technology. Nat Commun. 2019;10:1-9.

11. Kono N, Arakawa K. Nanopore sequencing: review of potential applications in functional genomics. Develop Growth Differ. 2019;61:316-26.

12. Di Resta C, Galbiati S, Carrera P, Ferrari M. Next-generation sequencing approach for the diagnosis of human diseases: open challenges and new opportunities. J Int Fed Clin Chem Lab Med. 2018;29:4-14.

13. Dingle TC, MacCannell DR. Molecular strain typing and characterisation of toxigenic Clostridium difficile. Methods in Microbiology. Elsevier Ltd. 2015:329-57.

14. Kapatai G, Sheppard CL, Al-Shahib A, Litt DJ, Underwood AP, Harrison TG, et al. Whole genome sequencing of Streptococcus pneumoniae: development, evaluation and verification of targets for serogroup and serotype prediction using an automated pipeline. PeerJ. 2016;4:e2477.

15. Mauffrey F, Fournier É, Demczuk W, Martin I, Mulvey M, Martineau C, et al. Comparison of sequential multiplex $\mathrm{PCR}$, sequetyping and whole genome sequencing for serotyping of Streptococcus pneumoniae. PLoS One. 2017;12:1-16.

16. Ardanuy C, Rolo D, Fenoll A, Tarrago D, Calatayud L, Liñares J. Emergence of a multidrug-resistant clone (ST320) among invasive serotype 19A pneumococci in Spain. J Antimicrob Chemother. 2009:64:507-10.

17. Enright MC, Spratt BG. A multilocus sequence typing scheme for Streptococcus pneumoniae: identification of clones associated with serious invasive disease. Microbiology. 1998;144:3049-60.

18. Hao W, Ma JH, Warren K, Tsang RSW, Low DE, Jamieson FB, et al. Extensive genomic variation within clonal complexes of Neisseria meningitidis. Genome Biol Evol. 2011;3(1):1406-18.

19. Koren S, Walenz BP, Berlin K, Miller JR, Bergman NH, Phillippy AM. Canu : scalable and accurate long-read assembly via adaptive $k$-mer weighting and repeat separation. Genome Res. 2017;27:722-36.

\section{Publisher's Note}

Springer Nature remains neutral with regard to jurisdictional claims in published maps and institutional affiliations.

Ready to submit your research? Choose BMC and benefit from:

- fast, convenient online submission

- thorough peer review by experienced researchers in your field

- rapid publication on acceptance

- support for research data, including large and complex data types

- gold Open Access which fosters wider collaboration and increased citations

- maximum visibility for your research: over $100 \mathrm{M}$ website views per year

At $\mathrm{BMC}$, research is always in progress.

Learn more biomedcentral.com/submissions 